\title{
ANATOMIA DO LENHO EM ESPÉCIES SUL-BRASILEIRAS DA SÉRIE STIPELLARES BENTH. (MIMOSA L. - FABACEAE). 1 - FUNDAMENTOS DE TAXONOMIA E ANATOMIA DA MADEIRA ${ }^{1}$
}

\author{
JOSÉ NEWTON CARDOSO MARCHIORI ${ }^{2}$ \\ PAULO FERNANDO DOS SANTOS MACHADO ${ }^{3}$
}

\section{RESUMO}

No presente artigo, que dá início a uma série dedicada ao estudo anatômico do lenho em espécies da Série Stipellares Benth. (Mimosa L. - Fabaceae), são analisadas questões taxonômicas e de anatomia da madeira, como embasamento à linha de pesquisa.

Palavras-chave: Anatomia da Madeira, Fabaceae, Mimosa, Mimosaceae, Stipellares, Taxonomia da madeira.

\section{ABSTRACT}

[Wood anatomy of Southern Brazilian species from Series Stipellares Benth. (Mimosa L. Fabaceae). 1 - Theoretical foundations from Taxonomy and Wood Anatomy].

In this article, which iniciates a series devoted to the anatomy of wood species from series Stipellares Benth. (Mimosa L. - Fabaceae), taxonomic and anatomic issues are analysed, as a basis to this line of research.

Key words: Fabaceae, Mimosa L., Mimosaceae, Stipellares Benth., Wood Anatomy, Wood Taxonomy.

\section{INTRODUÇÃO}

Apesar do elevado número de espécies na flora lenhosa sul-brasileira, o gênero Mimosa L. (Fabaceae: Mimosoideae) ainda é pouco conhecido no tocante à anatomia da madeira, carecendo de uma abordagem estrutural mais ampla, com vistas ao reconhecimento de táxones infragenéricos.

Ao propor a investigação do lenho em espécies da série Stipellares Benth., uma das mais representativas no sul do Brasil, a presente linha de pesquisa visa a suprir parte desta lacuna, seja pela descrição microscópica de lenhos ainda desconhecidos, seja pela realização de uma

1 Recebido em 05-12-2015 e aceito para publicação em 10-2-2016.

2 Engenheiro Florestal, Dr. Bolsista de Produtividade em Pesquisa (CNPq - Brasil), Professor Titular do Departamento de Ciências Florestais, Universidade Federal de Santa Maria. Santa Maria, RS, Brasil. marchiori@pq.cnpq.br

3 Engenheiro Florestal, doutorando do Programa de PósGraduação em Engenharia Florestal, Universidade Federal de Santa Maria. barcasole@gmail.com análise de agrupamento hierárquica aglomerativa, com base em caracteres anatômicos qualitativos e quantitativos da madeira. A escolha da referida série botânica justifica-se, ainda, pela diversidade de ambientes ocupados por suas espécies, aspecto que, ao lado de um enfoque taxonômico, também requer exame ecológico-estrutural, agregando interesse científico à pesquisa.

\section{REVISÃO DE LITERATURA}

A utilização de caracteres internos das plantas na classificação vegetal é muito antiga e tem sido utilizada com freqüência (Record, 1934). A esse respeito, Dadswell (1939) pondera que a anatomia da madeira pode contribuir na classificação de gêneros e famílias de posição taxonômica duvidosa, no estudo do arranjo interno de famílias e na determinação das prováveis afinidades entre famílias botânicas.

Em taxonomia da madeira é fundamental distinguir os caracteres anatômicos que são relativamente constantes, dos passíveis de variação sob diferentes condições de cres- 
cimento. Para isso, Rendle (1944) considera indispensável uma boa experiência para reconhecer os caracteres de valor diagnóstico em uma madeira e estimar seu valor como típico de família, gênero, de um táxon infragenérico ou espécie.

A presença de ornamentações em pontoações intervasculares, por exemplo, ocorre em famílias inteiras ou determinados grupos de uma família. Panshin \& De Zeeuw (1970), entre outros, reportam o caráter para todas as espécies norteamericanas da família Leguminosae (Fabaceae), com exceção de Cercis L. (Fabaceae: Caesalpinioideae). Por sua vez, o número, diâmetro e distribuição destas ornamentações podem variar consideravelmente e ter valor diagnóstico (Bailey, 1951; Ohtani, 1981; Ohtani et al., 1983; Vliet, 1978; Vliet \& Baas, 1984).

Outros caracteres, como arranjo e abundância de poros (poros $/ \mathrm{mm}^{2}$ ), comprimento de elementos vasculares $(\mu \mathrm{m})$ e fração de parênquima axial (\%), podem ser marcadamente influenciados pelo ambiente (Metcalfe \& Chalk, 1972).

No tocante aos raios, o caráter de maior utilidade é a largura, tanto em dimensão linear como em número de células; a altura também pode ser valiosa, principalmente se os valores forem ou muito altos ou muito baixos. A presença de raios exclusivamente unisseriados também se revela importante, podendo auxiliar na distinção de gêneros e espécies, sobretudo se o caráter tiver ocorrência esporádica no grupo em questão (Metcalfe \& Chalk, 1972).

O comprimento de elementos vasculares, porosidade (difusa ou em anel), vasos agrupados em cadeias ou solitários, presença (ou ausência) de canais intercelulares, tilos, tipos de parênquima axial e ocorrência de estratificação, também apresentam valor diagnóstico (Titmuss, 1948).

Nas últimas décadas, a associação de técnicas estereológicas e manipulação computadorizada de dados estatísticos proporcionaram uma notável contribuição para a identificação de madeiras e segregação de espécies afins, além de auxiliar na predição de propriedades físicomecânicas, comportamento tecnológico e uso potencial de madeiras (Marchiori, 1990). No mesmo sentido, a análise de agrupamento hierárquica aglomerativa tem contribuído - e de modo significativo -, para a taxonomia botânica, com as vantagens de seguir critério independente, avesso a subjetividades.

\section{Taxonomia no gênero Mimosa $\mathbf{L}$.}

Contemplada por dois estudos magistrais, distanciados entre si por um lapso de mais de cem anos, a taxonomia do gênero Mimosa L. foi pouco questionada ao longo do tempo e ainda resta à espera de eventual revisão, com base em estudos de genética molecular. Mesmo antes disso, todavia, novidades importantes podem surgir em campos insuficientemente investigados, caso da anatomia da madeira.

George Bentham, o mais importante leguminólogo do século XIX, organizou as espécies de Mimosa L. da flora brasileira em duas seções - Eumimosa DC. e Habbasia Benth. -, distintas entre si pela presença de flores haplostêmones e diplostêmones, respectivamente. Para a seção Eumimosa, Bentham (1876) reconheceu 12 séries: Sensitivae, Modestae, Castae, Pectinatae, Pudicae, Hirsutae, Pedunculosae, Meticulosae, Myriophyllae, Spiciflorae, Obstrigosae e Lepidotae. No caso da seção Habbasia, o taxonomista inglês segregou as espécies em 9 séries: Leptostachyae, Glanduliferae, Rubicaules, Stipellares, Leptopodae, Somnianthes, Asperatae, Adversae e Pachycarpae. De larga vigência, esse esquema predominou na literatura taxonômica por mais de cem anos.

Na última década do século vinte, Rupert A. Barneby propôs um novo esquema taxonômico, em alentado estudo sobre as espécies do continente americano. A partir de ancestrais Piptadenióides, a seção Mimadenia é a primeira em seu "hipotético" esquema filogenético, seguida pela seção Batocaulon, a qual teria dado origem às seções Calothamnos e Habbasia; a partir desta, por fim, ter-se-ia derivado a seção 
Mimosa (Barneby, 1991). Para cada uma destas cinco seções, o taxonomista do século XX reconheceu diversas séries botânicas. A respeito desse complexo esquema taxonômico, cabe salientar que alguns táxones infragenéricos propostos por Bentham foram aproveitados por Barneby, e que outros tantos foram especialmente criados pelo taxonomista angloamericano.

Tendo em vista o grande número de espécies de Mimosa na flora sul-rio-grandense, a presente linha de pesquisa restringe-se ao estudo anatômico das espécies lenhosas da série Stipellares Benth., pertencente à seção Batocaulon DC.

\section{A Série Stipellares Benth.}

Na Flora Brasiliensis, Bentham (1876) reconheceu 8 espécies na série Stipellares, referindo, para a mesma, os seguintes caracteres morfológicos: arbustos inermes ou com acúleos esparsos; capítulos globosos axilares; legumes sub-planos de valvas indivisas; estipelas orbiculares ou ovais; e folíolos pouco abundantes.

Barneby (1991) reconheceu 19 espécies válidas para o mesmo grupo, distribuídas no Brasil extratropical, Paraguai, centro-norte da Argentina e Uruguai, estendendo-se, além do Trópico de Capricórnio, apenas em São Paulo, no departamento de Amambay (Paraguai) e com uma espécie isolada na Bolívia (Mimosa lepidota), na encosta leste dos Andes.

Para o estado de Santa Catarina, Burkart (1979) citou apenas três espécies: Mimosa hassleriana (atual M. bifurca), M. trachycarpa e $M$. cruenta. No caso do Rio Grande do Sul, Rambo (1966) reconheceu seis espécies (Mimosa amphigena, M. bracteolaris, M. cruenta, $M$. hassleriana, $M$. intricata e $M$. iperoensis), número que foi ampliado por pesquisas de campo mais recentes.

\section{Anatomia da madeira em Fabaceae Mimosoideae}

Cronquist (1968) e Takhtajan (1958), dois dos mais importantes taxonomistas do século vinte, elevaram as subfamílias de Leguminosas à categoria de famílias botânicas. Na literatura anatômica, esta concepção taxonômica foi adotada em "Anatomy of the Dicotyledons", obra em que as tradicionais subfamílias de Leguminosae foram tratadas como famílias distintas (Mimosaceae, Caesalpiniaceae, Papilionaceae). A este respeito, resta salientar que Metcalfe \& Chalk (1972) reconheceram as Mimosaceae como menos evoluídas e Papinionaceae como mais especializadas.

Antes, mesmo, dessa clássica obra em anatomia da madeira, Record \& Hess (1949) e Senn (1943) já consideravam vantajosa a manutenção da unidade da família Leguminosae (atual Fabaceae), devido à inexistência de uma linha nítida de diferenciação entre suas subfamílias.

Em estudo anatômico sobre as Leguminosas da Argentina, Cozzo (1951) reconheceu que Mimosoideae e Caesalpinioideae são estruturalmente mais homogêneas do que Papilionoideae, sendo difícil o estabelecimento de diferenciações práticas entre suas tribos e gêneros. Segundo o autor, a subfamília Mimosoideae apresenta variação anatômica ainda menor do que Caesalpinioideae.

Segundo Metcalfe \& Chalk (1972), as Mimosaceae têm os raios mais especializados dos três grupos, pois são invariavelmente homogêneos, de células procumbentes e com unisseriados relativamente escassos. Os autores reforçam suas conclusões filogenéticas afirmando que, apesar do elevado e uniforme desenvolvimento radial das Mimosaceae, em Caesalpiniaceae e, sobretudo, em Papilionaceae, encontram-se raios ainda mais especializados, bem como tipos menos evoluídos. 
Baretta-Kuipers (1981), em estudo com 35 gêneros de Mimosoideae, 85 de Caesalpinioideae e 68 de Papilionoideae, comprovou que a estrutura dos raios é a característica anatômica mais importante para a taxonomia das Leguminosae, e que Caesalpinioideae apresenta a estrutura menos evoluída da família. Segundo a autora, esta subfamília constitui a base, a partir da qual a especialização anatômica seguiu dois rumos distintos: em direção às Mimosoideae, com a especialização dos raios, e em direção às Papilionoideae, com a estratificação de todos os elementos. É interessante notar que Burkart (1952) chegou, praticamente, à mesma conclusão, com base na morfologia externa.

Em estudo anatômico de alguns gêneros sul-americanos, Reinders-Gouwentak (1955) concluiu que pela "estrutura estratificada" convém dividir as Leguminosae em duas subfamílias - Mimosaceae e Papilionaceae , a última das quais incluindo Caesalpinioideae.

Para as Mimosoideae, na qual se insere Mimosa L., Baretta-Kuipers (1981) destaca que o lenho pode ser facilmente reconhecido pela ausência de estratificação completa e pelos raios sempre homocelulares, com células procumbentes de seção pequena em plano tangencial, baixas em plano radial. A autora refere que este último aspecto raramente se encontra em Caesalpinioideae, estando associado, neste caso, à estratificação de raios.

Para a identificação de tribos de Leguminosae, Baretta-Kuipers (1981) ressalta, como importantes, o tipo de raios (homocelulares ou heterocelulares), a presença de raios uni ou multisseriados e a ocorrência de estratificação. Para a autora, o arranjo do parênquima axial adquire valor na segregação de gêneros e espécies.

\section{Anatomia do lenho no gênero Mimosa $\mathbf{L}$.}

De acordo com Cozzo (1951), o lenho apresenta ampla variação anatômica no gênero Mimosa L., inexistindo uma característica peculiar ao mesmo, comum a todas as espécies. Embora pouco investigado, devido ao pequeno porte de grande parte de suas espécies lenhosas e o escasso o valor econômico da maioria das mesmas, a diversidade estrutural referida na literatura corresponde ao descrito para toda a subfamília Mimosoideae.

Um dos primeiros estudos deve-se a Record \& Hess (1949), autores que, baseados em Mimosa bracaatinga Hoehne (atual Mimosa scabrella Benth.) e Mimosa schomburgkii Benth. (espécie nativa da Guiana), referem, apenas, caracteres gerais e organolépticos do lenho. Cabe destacar que Mimosa L. não foi citada uma única vez no tocante a caracteres microscópicos. De igual modo, Metcalfe \& Chalk (1972) não fazem menção ao gênero Mimosa, em sua diagnose anatômica das Mimosaceae.

Em chave anatômica para 13 espécies argentinas de Mimosa, Cozzo (1951) relacionou os seguintes aspectos anatômicos para o mesmo:

- Anéis de crescimento escassamente definidos, melhor demarcados em Mimosa ostenii.

- Porosidade difusa, com tendência a circular em Mimosa ostenii, em arranjo dendrítico "bem notável" em $M$. hassleriana e $M$. uliginosa, e apenas tendente a esse arranjo em $M$. cruenta e M. uruguensis.

- Vasos solitários e em múltiplos de 2-3, menos comumente 4-5 poros; solitários e em múltiplos de 2 em Mimosa scabrella e $M$. adpressa; compondo longos múltiplos nas espécies com porosidade dendrítica.

- Vasos de seção oval, angulares e de paredes delgadas em Mimosa pigra, espessas em $M$. polycarpa.

- Elementos vasculares retilíneos ou levemente sinuosos, com placas de perfuração simples, oblíquas.

- Apêndices ausentes ou curtos, por vezes longos.

- Pontoações intervasculares ornamentadas, alternas, pequenas a médias, grandes em Mimosa pigra. 
- Pontoações raio-vasculares e parênquimovasculares, semelhantes às intervasculares; semiareoladas em Mimosa pigra.

- Tilos e espessamentos espiralados, geralmente ausentes; espessamentos delgados, em elementos muito curtos de Mimosa cruenta.

- Traqueóides vasculares, escassos, em espécies de porosidade dendrítica.

- Raios heterogêneos a quase homogêneos; exclusivamente unisseriados em Mimosa pigra, predominantemente unisseriados em $M$. uruguensis, geralmente trisseriados em $M$. adpressa, com multisseriados estreitos (até 5 células) em $M$. bimucronata e $M$. polycarpa.

- Raios com células envolventes curtas em algumas espécies, com células radiais oleosas em $M$. bimucronata.

- Parênquima axial abundante ou muito abundante em Mimosa cruenta, M. detinens e $M$. hassleriana, menos desenvolvido em $M$. adpressa, M. bimucronata e M. scabrella; no primeiro grupo, do tipo paratraqueal-em-faixas, comumente curto-confluente.

- Células parenquimáticas axiais em séries e fusiformes, com predomínio de fusiformes em M. pigra.

- Fibras libriformes muito curtas, de paredes finas a semi-espessas, frequentemente septadas; fibrotraqueóides, em Mimosa polycarpa.

- Estratificação incompleta, uniforme, em Mimosa adpressa e M. scabrella (Cozzo, 1951)

No gênero em estudo, a espécie de madeira mais investigada é Mimosa scabrella Benth. (Tortorelli, 1956; Richter \& Charvet, 1973; Marchiori, 1995), mercê de seu interesse florestal. Da mesma seção Batocaulon DC. e série Calothamnos Barneby, dispõe-se, igualmente, de descrições anatômicas de Mimosa berroi Burkart (Marchiori, 1996a), Mimosa daleoides Benth. (Marchiori, 1982), Mimosa eriocarpa Benth. (Carnieletto \& Marchiori, 1993), Mimosa incana (Marchiori, 1996c), Mimosa flocculosa Burkart (Pereira et al., 2013) e Mimosa pilulifera Benth. (Marchiori \& Muñiz, 1997).
Com relação a outras séries botânicas, foram microscopicamente descritos os lenhos de Mimosa micropteris Benth. (Siegloch et al, 2013), Mimosa sparsa Benth. (Maccari \& Marchiori, 1994) e Mimosa bimucronata (DC.) Kuntze (Marchiori, 1993).

Com relação à série Stipellares Benth., objeto da presente pesquisa, foram anatomicamente descritos os lenhos de Mimosa cruenta (Marchiori, 1985) e Mimosa uraguensis (Marchiori, 1996b). Em sua "chave para o reconhecimento anatômico dos gêneros e espécies de Mimosoídeas e Caesalpinioídeas argentinas", Cozzo (1951) assinalou a presença de porosidade dendrítica em Mimosa cruenta, $M$. hassleriana (atual $M$. bifurca) e $M$. uraguensis, e poros agrupados, "em sua maioria", em Mimosa uliginosa. Apesar da não citação do caráter para espécies de outras séries botânicas, o autor não tece qualquer comentário a respeito de eventual vinculação taxonômica do mesmo.

\section{REFERÊNCIAS BIBLIOGRÁFICAS}

BAILEY, I.W. The use and abuse of anatomical data in the study of phylogeny and classification. Phytomorphology, v. 1, p. 67-69, 1951.

BARETTA-KUIPERS, T. Wood anatomy of Leguminosae: its relevance to Taxonomy. In: POLHILL, R.M.; RAVEN, P.H. Advances to Legume Systematics. 1981. p. 677-715.

BARNEBY, R.C. Sensitivae Censitae: a description of the genus Mimosa Linnaeus (Mimosaceae) in the New World. Mem. New York Botanical Gardens, v. 65, p. 1-835, 1991.

BENTHAM, Leguminosae. III. Mimoseae. In: MARTIUS, C.F.O. von. Flora Brasiliensis, v. 15 , n. 2, p. 259-527, 1876.

BURKART, A. Las leguminosas argentinas silvestres y cultivadas. Buenos Aires: ACME, 1952. 569p.

BURKART, A. Leguminosas Mimosoideas. In: REITZ, R. Flora Ilustrada Catarinense. Itajaí: Herbário Barbosa Rodrigues, 1979. 299 p.

CARNIELETTO, C.; MARCHIORI, J.N.C. Anatomia da madeira de Mimosa eriocarpa Benth. Ciência Florestal, Santa Maria, v. 3, n. 1, p. 107-120, 1993. 
COZZO, D. Anatomia del leño secundario de las Leguminosas Mimosoideas y Cesalpinoideas argentinas silvestres y cultivadas. Rev. Inst. Nac. Invest. Ci. Nat. Ci. Bot., Buenos Aires, v. 2, n. 2, p. 63-290, 1951.

CRONQUIST, A. The evolution and classification of flowering plants. Boston: Houghton Mifflin, 1968. 396 p.

DADSWELL, H.E. The role of Wood Anatomy in Forest Botany. J. Counc. Sci. Indust. Res. Aust., v. 12, p. 237-242, 1939.

MACCARI, A.; MARCHIORI, J.N.C. Estudo anatômico do xilema secundário de Mimosa sparsa Benth. Ciência Florestal, Santa Maria, v. 4, n. 1, p. 145-155, 1994.

MARCHIORI, J.N.C. A estrutura do xilema secundário de Mimosa daleoides Benth. (Leguminosae Mimosoideae). Ciência e Natura, Santa Maria, n. 4, p. 107-113, 1982.

MARCHIORI, J.N.C. Anatomia da madeira de Mimosa cruenta Benth. (Leguminosae Mimosoideae). Ciência e Natura, Santa Maria, n. 7, p. 73-81, 1985.

MARCHIORI, J.N.C. Anatomia das madeiras do gênero Acacia, nativas e cultivadas no estado do Rio Grande do Sul. Curitiba: UFPR, 1990. $226 \mathrm{f}$. Tese (Doutorado em Engenharia Florestal - Universidade Federal do Paraná).

MARCHIORI, J.N.C. Anatomia da madeira e casca do maricá, Mimosa bimucronata (DC.) O. Kuntze. Ciência Florestal, Santa Maria, v. 3, n. 1, p. 85-106, 1993.

MARCHIORI, J.N.C. Anatomia da madeira e casca da bracatinga, Mimosa scabrella Benth. Ciência e Natura, Santa Maria, n. 17, p. 115-132, 1995.

MARCHIORI, J.N.C. Anatomia do xilema secundário de Mimosa berroi Burk. Ciência e Natura, Santa Maria, n. 18, p. 117-129, 1996 a.

MARCHIORI, J.N.C. Anatomia do xilema secundário de Mimosa uraguensis Hook. \& Arn. Ciência e Natura, Santa Maria, v. 18, p. 103115, $1996 \mathrm{~b}$.

MARCHIORI, J.N.C. Anatomia do xilema secundário de Mimosa incana (Spreng.) Benth. Ciência Florestal, Santa Maria, v. 6, n. 1, p. 5363, 1996c.

MARCHIORI, J.N.C.; MUÑIZ, G.I.B. de. Estudo anatômico do xilema secundário de Mimosa pilulifera Benth. Ciência Florestal, Santa Maria, v. 7, n. 1, p. 65-75, 1997.

METCALFE, C.R.; CHALK, L. Anatomy of Dicotyledons. Oxford: Clarendon Press, 1972. $1500 \mathrm{p}$.

OHTANI, J. Vesture or warts. Proposed terminology. IAWA Bulletin, v. 5, n. 1, p. 37-40, 1981.

OHTANI, J.; MEYLAN, B.A.; BUTTERFIELD, B.G. Occurrence of warts in the vessel elements and fibres of New Zealand woods. New Zealand Journal of Botany, n. 21, p. 359-372, 1983.

PANSHIN, A.J.; DE ZEEUW, C. Textbook of Wood Technology. New York: McGraw-Hill, 1970. v. 1. $705 \mathrm{p}$.

PEREIRA, A.C. da S.; SIEGLOCH, A.M.; MARCHIORI, J.N.C. Anatomia do lenho de Mimosa flocculosa Burkart. Balduinia, Santa Maria, n. 32, p. 29-33, 2013.

RAMBO, B. Leguminosae riograndenses. Pesquisas, São Leopoldo, Série Botânica, n. 23, p. 1-166, 1966.

RECORD, S.J. Role of the Wood Anatomy in Taxonomy. Tropical Woods, n. 37, p. 1-9, 1934.

RECORD, S.J.; HESS, R.W. Timbers of the New World. New Haven: Yale University Press, 1949. $640 \mathrm{p}$.

REINDERS-GOUWENTAK, C.A. The storied structure features and the taxonomic rank of the leguminous taxa. Acta Botanica Neerlandica, $\mathrm{n}$. 4, p. 460-470, 1955.

RENDLE, B.J. On the taxonomic value of the anatomical structure of the vegetative organs of Dicotyledons. 3. The role of anatomy in the practical identification of commercial timbers. Proceedings of the Linnean Society of London, v. 155, n. 3, p. 218-221, 1944.

RICHTER, H.G.; CHARVET, L.M. Estudo e pesquisa sobre Mimosa scabrella. Floresta, Curitiba, v. 4, n. 2, p. 68-69, 1973.

SENN, H.A. The relation of Anatomy and Cytology to the classification of the Leguminosae. Chron. Bot., n. 7, p. 306-308, 1943.

SIEGLOCH, A.M.; MARCHIORI, J.N.C.; SANTOS, S.R. dos. Anatomia do lenho de Mimosa micropteris Benth. Balduinia, Santa Maria, n. 40, p. 18-22, 2013.

TAKHTAJAN, A.L. Flowering plants: origin and dispersal. Edinburgh: Oliver \& Boyd, 1969. 310p. 
TORTORELLI, L.A. Maderas y bosques argentinos. Buenos Aires, ACME, 1956. 910p.

TITMUS, F.H.A. Concise encyclopaedia of world timbers. London: Mechanical Press, 1948. $156 \mathrm{p}$.

VLIET, G.J.C.M. van. The vestured pits of
Combretaceae and allied families. Acta Botanica Neerlandica, n. 27, p. 273-285, 1978.

VLIET, G.J.C.M. van; BAAS, P. Wood anatomy and classification of the Myrtales. Annals of the Missouri Botanical Garden, n. 71, p. 783-800, 1984. 